\title{
Evaluación formativa y aprendizaje de la técnica del remate de futbol de los estudiantes del nivel universitario
}

\section{Formative evaluation and learning of the technique of the auction of soccer of the Students Of The University Level}

\author{
Julio Cesar Suarez Sotelo', Abelardo Rodolfo Campana Concha²
}

\section{RESUMEN}

Objetivo: Determinar el grado de relación que tiene la evaluación formativa y el Aprendizaje de la técnica de remate de la asignatura de Futbol de los estudiantes del 6 to ciclo de la E.P.E.F. de la Facultad de Educación de la UNMSM, 2019. Material y Métodos: La investigación de tipo cuantitativa de diseño correlacional. Se aplicó un cuestionario y test a 46 estudiantes, cuyos resultados fueron procesados por el programa SPSS, del mismo modo siguiendo el procedimiento estadístico de Rho de Spearman. Resultado: La hipótesis general halló una correlación de Rho $=0.818$ que explica que la evaluación formativa aplicada de a veces tiene un vínculo relacional con el aprendizaje de la técnica de remate de futbol que alcanza un nivel regular. En cuanto a las cinco hipótesis específicas se encontró una relación de Rho $=0.492, \mathrm{Rho}=0.722, \mathrm{Rho}=0.514, \mathrm{Rho}=0,582$ y Rho $=$ 0,640 , respectivamente, lo cual se entiende que la forma de evaluación reguladora, procesual, continua, retroalimentadora e innovadora, incide en el aprendizaje de este curso. Conclusiones: la forma en la que el docente aplica su evaluación limita a las calificaciones del curso, ya que los estudiantes aún no son altos.

Palabras clave: Evaluación Formativa, Reguladora, Procesual, Continua, Retroalimentadora, Innovadora, Aprendizaje, Técnica, Remate de Fútbol

\begin{abstract}
Objective: To find out the intensity of connection between the formative evaluation and the learning of How to hit a Soccer Ball of the football subject of the students 6 th cycle E.P.E.F Education Faculty of the UNMSM, 2019. Material and Methods: The research of quantitative type of correlational design. A questionnaire and test were applied to 46 students, whose results were processed by the SPSS program, in the same way following the statistical procedure of Rho de Spearman. Result: The general hypothesis found a correlation of Rho $=0.818$ that explains that the applied formative assessment of sometimes has a relational link with the learning of the soccer spike technique that reaches a regular level. Regarding the five specific hypotheses, a relationship of $\mathrm{Rho}=0.492$, $\mathrm{Rho}=0.722$, $\mathrm{Rho}=0.514, \mathrm{Rho}=0.582$ and Rho $=0.640$, respectively, was found, which is understood to be the form of regulatory, procedural, continuous, and feedback evaluation. and innovative, affects the learning of this course. Conclusions: the way in which the teacher applies his evaluation limits the grades of the course, since the students are not yet high.
\end{abstract}

Keywords: Formative Evaluation, Regulatory, Procedural, Continuous, Feedback, Innovative, Learning, Technique, Soccer Shot

\section{INTRODUCCIÓN}

En la actualidad resulta una preocupación de cada docente conocer diferentes formas de evaluar a nuestros estudiantes, muchas veces se ve la evaluación como un factor netamente cuantitativo sin darle una interpretación, o se confunde con medición lo cual es una concepción totalmente errónea.

En la carrera de Educación Física de la UNMSM, se evalúa a los alumnos de la asignatura de Fútbol II, mediante 2 evaluaciones escritas (2), prácticas calificadas (1), presentación personal, asistencias (1) y la entrega de 1 carpeta (1). Las 5 notas se suman y se dividen entre 5 , este resultado sería la nota final del bimestre. Lo cual da un mayor porcentaje a las evaluaciones escritas. Creemos que esta forma de evaluar representa un modelo tradicional, ya que se centra más en el resultado que en el proceso y no permite que el alumno obtenga un adecuado aprendizaje.

Hernández (2019) sustenta que "las características del sistema de evaluación muchas veces son incoherentes con los propósitos de formación, por lo tanto la evaluación será un importante factor que condiciona el desarrollo de los aprendizajes, y competencias de los estudiantes y la promoción de la calidad educativa, alejándose de garantizar la congruencia y eficacia del sistema en su conjunto".

Por lo que menciona el autor, la forma de evaluar de muchos docentes no guarda relación con el propósito de formación además que es un condicionante en el desarrollo de los aprendizajes y competencias del estudiante.

El aprendizaje de diversas asignaturas que se imparte en la E.P.E.F. es un aspecto sumamente importante para la formación de futuros docentes en Educación Física. Esta al ser un área teórico-práctico, el estudiante deberá finalizar el curso con los conocimientos claros de técnicas y estrategias para poder tener un buen desempeño como docente en el ambiente educativo. Sin embargo, es importante plantear si las formas de evaluación en educación física son las adecuadas o pertinentes para para el logro de los aprendizajes.

El docente como mediador de este proceso que tiene lugar en el campo o aula debe tener conocimiento claro de no limitar o sesgar a la evaluación a un hecho tecnicista o de rendimiento, influenciado por corrientes conductistas, ya que sería una práctica problemática, teniendo en cuenta la diversificación de estudiantes que ingresan a las escuelas de formación en 
Educación Física además la evaluación de acciones motrices propias de un determinado deporte requiere de una atención especial por parte del docente por su naturaleza práctica donde se desarrollan capacidades físicas y técnicas.

La evaluación es un componente importante del proceso de enseñanza aprendizaje, no es un aspecto aislado; evaluar es atribuir valor a las cosas, es afirmar algo sobre su mérito. Se evalúa para comprender, alcanzar un cambio y mejorar.

Por otro lado, la evaluación formativa tiene por interés conseguir información relacionada alcances y problemas de los estudiantes, evitando el llamado de atención sobre sus notas, de esta manera poder adaptar la forma de enseñanza y sus elementos didácticos para mejores resultados.

Lograr que nuestros estudiantes generen nuevos aprendizajes es también un factor que como docentes tenemos que trabajar por ello es necesario que se plantee la siguiente problemática.

Este estudio se fundamenta en los estudios anteriores como los de Acevedo (2012) entienden la importancia de la evaluación para los buenos resultados académicos y los proceso de enseñanza. Por otro lado, sobre técnicas de futbol, tenemos a Espinoza y Pando (2007) quienes evidencian que estos influyen para perfeccionar los fundamentos técnicos del fútbol, por lo cual requieren de una guía de entrenamiento especializada en fútbol femenino, además de llevar una estadística de progreso de cada fundamento técnico en las señoritas deportistas.

El objetivo principal de la investigación es determinar el grado de relación que tiene las variables en los estudiantes del 6 to ciclo de la E.P.E.F. de la Facultad de Educación de la UNMSM, 2019, del mismo modo, se intenta explicar la relación entre las dimensiones reguladora, procesual, continua, retroalimentadora e innovadora, y la segunda variable.

\section{MATERIALES Y MÉTODOS}

Con respecto a los planteamientos de investigación se consideró el enfoque cuantitativo de nivel explicativo para establecer la relación de las variables, para la cual se aplicó un cuestionario y test de remate, respectivamente. Los resultados dependerán de los resultados de estos instrumentos.

\section{RESULTADOS}

Estos dependerán del procesamiento de la información en el programa SPSS, con el cual se estableció la contratación de la hipótesis general y específica, los cuales se describen a continuación:

\section{Hipótesis General}

HGA. Existe relación entre la evaluación formativa y el aprendizaje de la técnica de remate en la asignatura de futbol de los estudiantes de la E.P.E.F. U.N.M.S.M, 2019.

HGO. No existe relación entre la evaluación formativa y el aprendizaje de la técnica de remate de la asignatura de futbol de los estudiantes de la E.P.E.F. U.N.M.S.M, 2019.
Tabla 1. correlación hipótesis general

\begin{tabular}{llll}
\hline correlacion & \multicolumn{2}{c}{ Aprendizaje de técnica del remate } \\
\hline & & $\begin{array}{l}\text { Coeficiente de } \\
\text { correlacion }\end{array}$ &, 818 \\
\cline { 3 - 3 } $\begin{array}{l}\text { Rho de } \\
\text { Spearman }\end{array}$ & $\begin{array}{l}\text { Evaluación } \\
\text { formativa }\end{array}$ & Sig.(bilitaral) &, 000 \\
\cline { 3 - 4 } & $\mathrm{N}$ & 46 \\
\hline
\end{tabular}

El índice de correlación es 0,818 muy buena, se confirma la hipótesis alterna, estableciéndose en a veces la evaluación formativa, relacionado al aprendizaje de nivel regular de la técnica de remate de fútbol.

\section{Específica $N^{\circ} 1$}

Ha. Existe relación entre la acción reguladora y el aprendizaje de la técnica de remate de la asignatura de futbol de los estudiantes del nivel superior.

Ho. No existe relación entre la acción reguladora y el aprendizaje de la técnica de remate de la asignatura de futbol de los estudiantes del nivel superior.

Tabla 2. Correlación hipótesis específica 1

\begin{tabular}{|c|c|c|c|}
\hline correlacion & & Aprendizaje de & remate \\
\hline \multirow{3}{*}{$\begin{array}{l}\text { Rho de } \\
\text { Spearman }\end{array}$} & \multirow{3}{*}{ Reguladora } & $\begin{array}{l}\text { Coeficiente de } \\
\text { correlacion }\end{array}$ &, $492^{* *}$ \\
\hline & & Sig.(bilitaral) & ,001 \\
\hline & & $\mathrm{N}$ & 46 \\
\hline
\end{tabular}

La correlación es 0,492 moderado, por lo tanto validaría la hipótesis específica alterna. Establece la aplicación de casi siempre en la evaluación reguladora está relacionado al aprendizaje de nivel regular en la técnica de remate de fútbol.

\section{Específica $\mathrm{N}^{\circ} 2$}

Ha. Existe relación significativa entre la acción procesual y el aprendizaje de la técnica de remate de los estudiantes del nivel superior.

Ho. No existe relación significativa entre la acción procesual y el aprendizaje de la técnica de remate de los estudiantes del nivel superior.

Tabla 3 Correlación hipótesis específica 2

\begin{tabular}{|c|c|c|c|}
\hline correlacion & & Aprendizaje de & remate \\
\hline \multirow{3}{*}{$\begin{array}{l}\text { Rho de } \\
\text { Spearman }\end{array}$} & \multirow{3}{*}{ Procesual } & $\begin{array}{l}\text { Coeficiente de } \\
\text { correlacion }\end{array}$ &, $514^{* *}$ \\
\hline & & Sig.(bilitaral) & ,000 \\
\hline & & $\mathrm{N}$ & 46 \\
\hline
\end{tabular}


BIG BANG 2021 Vol. 10(2)

Podemos apreciar que presenta un índice de correlación de 0,722 correspondiente a un grado bueno, por lo tanto se valida la hipótesis específica alterna y significaría también que hay evidencia estadística suficiente para rechazar la hipótesis nula. Asimismo, se establece que la aplicación de a veces en la evaluación procesual está relacionado al nivel regular del aprendizaje de la técnica de remate de fútbol.

\section{Específica $\mathrm{N}^{\circ} 3$}

Ha. Existe relación entre la acción continua y aprendizaje de la técnica de remate del futbol de los estudiantes del nivel superior.

Ho. No existe relación entre la acción continua y aprendizaje de la técnica de remate futbol de los estudiantes del nivel superior.

Tabla 4

Correlación hipótesis especifica 3

\begin{tabular}{|c|c|c|c|}
\hline correlacion & & \multicolumn{2}{|c|}{ Aprendizaje de técnica del remate } \\
\hline \multirow{3}{*}{$\begin{array}{l}\text { Rho de } \\
\text { Spearman }\end{array}$} & \multirow{3}{*}{ Continua } & $\begin{array}{l}\text { Coeficiente de } \\
\text { correlacion }\end{array}$ &, $514^{* *}$ \\
\hline & & Sig.(bilitaral) &, 000 \\
\hline & & $\mathrm{N}$ & 46 \\
\hline
\end{tabular}

El índice de correlación resultó 0,514 moderado, valida la hipótesis específica alterna, se establece en casi siempre la evaluación continua en relacion nivel regular del aprendizaje de la técnica de remate de fútbol.

\section{Específica $\mathrm{N}^{\circ} 4$}

Ha. Existe relación significativa entre la retroalimentación y el aprendizaje de la técnica de remate de la asignatura de fútbol de los estudiantes del nivel superior

Tabla 5. Correlación hipótesis específica 4

\begin{tabular}{|c|c|c|c|}
\hline correlacion & & Aprendizaje de & remate \\
\hline \multirow{3}{*}{$\begin{array}{l}\text { Rho de } \\
\text { Spearman }\end{array}$} & \multirow{3}{*}{ Retroalimentadora } & $\begin{array}{l}\text { Coeficiente de } \\
\text { correlacion }\end{array}$ &, $582^{\star \star}$ \\
\hline & & Sig.(bilitaral) &, 000 \\
\hline & & $\mathrm{N}$ & 46 \\
\hline
\end{tabular}

Existe una correlación de 0,582 moderado, confirmando hipótesis específica alterna. La opinión es a veces de la evaluación retroalimentadora en relacion al regular del aprendizaje de la técnica de remate de fútbol.

\section{Específica $\mathrm{N}^{\circ} 5$}

Ha. Existe relación significativa entre la acción innovadora y el aprendizaje de la técnica de remate de los estudiantes del nivel superior.

Ho. No existe relación significativa entre la acción innovadora y el aprendizaje de la técnica de remate de los estudiantes del nivel superior.

Tabla 6. Correlación hipótesis específica 5

\begin{tabular}{|c|c|c|c|}
\hline correlacion & & Aprendizaje de & remate \\
\hline \multirow{3}{*}{$\begin{array}{l}\text { Rho de } \\
\text { Spearman }\end{array}$} & \multirow{3}{*}{ Innovadora } & $\begin{array}{l}\text { Coeficiente de } \\
\text { correlacion }\end{array}$ &, $640^{* *}$ \\
\hline & & Sig.(bilitaral) & ,000 \\
\hline & & $\mathrm{N}$ & 46 \\
\hline
\end{tabular}

Se ve correlación de 0,640 se valida la hipótesis específica alterna. Asimismo, se establece como a veces la evaluación innovadora relacionada al nivel regular del aprendizaje de la técnica de remate de fútbol.

\section{DISCUSIÓN}

La evaluación formativa es un pilar fundamental hoy en la educación, definitivamente ayuda a desarrollar mejor los aprendizajes, la toma de decisiones, influye notablemente en la retroalimentación y dinamiza el hecho educativo. Sin embargo existe una gran preocupación por el bajo interés y la poca responsabilidad que algunos docentes tienen respecto a la evaluación formativa.

El objetivo de este trabajo fue demostrar la relación de las variables planteadas para esta investigación, en los estudiantes del nivel superior tomando en cuenta la aplicación de un instrumento de recolección de datos como lo fue la encuesta hacia los alumnos así como la toma de un test de aplicación de la técnica de remate.

El análisis de la hipótesis general se encontró una relación muy buena de 0.818 , lo cual indica que para la variable de evaluación formativa la mayoría de estudiantes marco la opción a veces, con un

$60.9 \%$ del total. Por otra parte, en los resultados del aprendizaje de la técnica de remate la mayoría de alumnos se clasifican en el grupo de regulares con un $71,7 \%$ del total. Con ello se alerta que a perspectiva de los estudiantes, la relativa frecuencia en la que forma en la que enseña el docente se enlaza a la ausencia de calificaciones muy destacadas en el curso en cuestión.

En la hipótesis específica 1, se halló una relación moderada, lo cual indica que para la evaluación reguladora la mayoría de estudiantes calificó de casi siempre con un $45.7 \%$ del total. Por otro lado, en los resultados del aprendizaje de la técnica de remate la mayoría de alumnos se clasifican en el grupo de regulares con un $71,7 \%$ del total. Con ello se expresa que a opinión de los estudiantes, la forma en la que el docente planifica las actividades y programa los contenidos de clase se enlazan a la ausencia de calificaciones muy destacadas en el curso en cuestión.

En la hipótesis específica 2, se halló una relación buena, indica que para la evaluación procesual la mayoría de estudiantes lo señaló de a veces con un $60.9 \%$ del total. Por otro lado, en los resultados del aprendizaje de la técnica de remate la mayoría de alumnos se clasifican en el grupo de regulares con un $71,7 \%$ 
del total. Con ello se expresa que a opinión de los estudiantes, la relativa frecuencia en la que forma en la que el docente motiva y mide saberes previos sobre el tema se enlaza a la ausencia de calificaciones muy destacadas en el curso en cuestión.

En la hipótesis específica 3 se identificó una relación moderada, lo cual indica que para la evaluación continua la mayoría de estudiantes lo señaló de casi siempre con un $43.5 \%$ del total. Por otro lado, en los resultados del aprendizaje de la técnica de remate la mayoría de alumnos se clasifican en el grupo de regulares con un $71,7 \%$ del total. Con ello se expresa que a opinión de los estudiantes, la frecuencia en la que forma en la que el docente resuelve dudas evaluativas, desarrolla aspectos teóricos y prácticos, y motiva a mejorar resultados incide en la ausencia de calificaciones muy destacadas en el curso en cuestión.

En la hipótesis específica 4 se identificó una relación moderada, lo cual indica que para la evaluación retroalimentadora la mayoría de estudiantes lo señaló de a veces con un $52.2 \%$ del total. Por otra parte, en los resultados del aprendizaje de la técnica de remate la mayoría de alumnos se clasifican en el grupo de regulares con un $71,7 \%$ del total. Con ello se expresa que a opinión de los estudiantes, la relativa frecuencia en la que forma en la que el docente reflexiona los resultados académicos, explica temas complicados y replantea aspectos evaluativos se enlaza a la ausencia de calificaciones muy destacadas en el curso en cuestión.

En la hipótesis específica 5 se identificó una relación moderada, lo cual indica que para la evaluación innovadora la mayoría de estudiantes lo señaló de a veces con un $60.9 \%$ del total. De otro lado, en los resultados del aprendizaje de la técnica de remate la mayoría de alumnos se clasifican

en el grupo de regulares con un $71,7 \%$ del total. Con ello se expresa que a opinión de los estudiantes, la relativa frecuencia en la que forma en la que el docente fomenta la participación, la utilización de instrumentos innovadores y la interacción en dinámicas grupales se enlaza a la ausencia de calificaciones muy destacadas en el curso en cuestión.

En el presente trabajo se ha podido contrastar la afirmación de Tarazona (2011) el cual concluye en que la evaluación para formación desarrollado en el proceso educativo confirma una influencia positiva porque mejora el rendimiento académico del alumnado de la población en la que se desarrolló la investigación,. En otras términos, podemos señalar que si evaluación formativa que se da de manera permanente favorece una mejoría en el rendimiento, de igual forma en esta investigación si se hubiera tenido en cuenta esa lógica los estudiantes de la E.P.E.F. hubieran alcanzado mejores promedios en la ejecución de la técnica de remate.

Así mismo es importante también discutir la opinión de Matute y Muriel (2014) el cual establece que la evaluación en el desarrollo del proceso de aprendizaje lo direcciona al análisis "sobre las estrategias e instrumentos implementados en el aula con el fin de adaptarlos a los progresos y necesidades observadas en el proceso de aprendizaje de los estudiante, así mismo la implementación de la evaluación formativa en los procesos de aprendizaje genera en los estudiantes una actitud más reflexiva frente a su propio aprendizaje". Por lo tanto se debe considerar instrumentos de evaluación formativa innovadores los cuales fortalezcan el proceso de de adquisición de nuevos conocimientos en los estudiantes de la EPEF.

\section{CONCLUSIONES}

1.Se evidenció la relación en la propuesta de la hipótesis general, lo cual se explica en que la frecuencia de a veces de la evaluación formativa se involucra en el aprendizaje de la técnica de remate de futbol a nivel regular. Concluyendo que la forma en la que el docente aplica su evaluación limita a las calificaciones del curso, ya que los estudiantes aún no son altas.

2.Se comprobó que la relación de la hipótesis específica 1, lo cual se explica en que la frecuencia de casi siempre de la evaluación reguladora se involucra en el aprendizaje de la técnica de remate de futbol a nivel regular. Concluyendo que la forma en la que el docente planifica las actividades y programa los contenidos de clase para evaluar a la clase limita a las calificaciones del curso, ya que los estudiantes aún no son altas.

3.Se encontró que la relación de la hipótesis específica 2 , lo cual se explica en que la frecuencia de a veces de la evaluación procesual se involucra en el aprendizaje de la técnica de remate de futbol a nivel regular. Concluyendo que la manera en la que el docente el docente motiva y mide saberes previos sobre el tema limita para alcanzar mejores calificaciones del curso, ya que los estudiantes aún no son altas.

4.Se determinó que la relación de la hipótesis específica 3 , lo cual se refiere a que la frecuencia de casi siempre de la evaluación continua se involucra en el aprendizaje de la técnica de remate de fútbol a nivel regular. Concluyendo que la manera en la que el docente resuelve dudas evaluativas, desarrolla aspectos teóricos y prácticos, y motiva a mejorar resultados incide, limita a los estudiantes para alcanzar mejores calificaciones del curso, ya que estos aún no son altas.

5.Se estableció que la relación de la hipótesis específica 4 , lo cual se refiere a que la frecuencia de casi siempre de la evaluación retroalimentadora se involucra en el aprendizaje de la técnica de remate de fútbol a nivel regular. Concluyendo que la manera en la que el docente reflexiona los resultados académicos, explica temas complicados y replantea aspectos evaluativos, limita a los estudiantes para alcanzar mejores calificaciones del curso, ya que estos aún no son altas.

6.Se halló que la relación de la hipótesis específica 5, lo cual se refiere a que la frecuencia de casi siempre de la evaluación innovadora se involucra en el aprendizaje de la técnica de remate de fútbol a nivel regular. Concluyendo que la manera en la que el docente fomenta la participación, utiliza instrumentos innovadores e incentiva la interactuación de dinámicas 
grupales, limita a los estudiantes para alcanzar mejores calificaciones del curso, ya que estos aún no son altas.

\section{AGRADECIMIENTOS}

A mi querida Escuela de Educación Física forjadora de grandes maestros

\section{REFERENCIAS BIBLIOGRÁFICAS}

Acevedo, I. (2012). La evaluación Formativa desde la Perspectiva del Maestro y su Impacto en el Rendimiento Académico de Alumnos en una Institución de Educación Superior en Medellín. Colombia: Edición Única.

Espinoza, J. y Pando, H. (2007). Guía de entrenamiento para perfeccionar los fundamentos técnicos del

fútbol en la selección de la Institución Educativa Femenino "Micaela Bastidas" de El Tambo - Huancayo (Tesis Pregrado). Universidad Nacional del Centro del Perú, Huancayo, Perú.

Hernández, G. (2019). Competencia docente y su relación con la evaluación formativa en la Facultad de Educación de la Universidad Nacional Mayor de San Marcos (Tesis Posgrado). UNMSM, Lima, Perú.

Matute, A y Muriel, L (2014) La evaluación formativa en los procesosde aprendizaje de $m$ ate mátic as (Tesis Posgrado). Universidad de Antioquia, Colombia. Recuperado de http://ayura.udea.edu.co:8080/jspu i/bitstream/123456789/1322/1/JC0 937.pdf.

Tarazona, J. (2011). Influencia de la evaluación formativa en el rendimiento académico de los estudiantes de la Escuela Profesional de Educación de la Universidad Nacional Santiago Antúnez de Mayolo (Tesis Posgrado). UNMSM, Lima, Perú. 